\title{
The effects of an 8-week stabilization exercise program on lumbar movement sense in patients with low back pain
}

\author{
Jean-Alexandre Boucher ${ }^{1,5,6^{*}}$, Richard Preuss ${ }^{2,6}$, Sharon M. Henry ${ }^{3}$, Jean-Pierre Dumas ${ }^{4}$ and Christian Larivière ${ }^{5,6}$
}

\begin{abstract}
Background: Lumbar stabilization exercises have gained popularity and credibility in patients with non-acute low back pain. Previous research provides more support to strength/resistance and coordination/stabilisation programs. Some authors also suggest adding strength/resistance training following motor control exercises. However, the effect of such a lumbar stabilization program on lumbar proprioception has never been tested so far. The present study investigated the effects of an 8-week stabilization exercise program on lumbar proprioception in patients with low back pain (LBP) and assessed the 8-week test-retest reliability of lumbar proprioception in control subjects.

Methods: Lumbar proprioception was measured before and after an 8-week lumbar stabilization exercise program for patients with LBP. Control subjects participated in the same protocol but received no treatment.

Results: The lumbar proprioception measure showed moderate reliability. Patients with LBP and control subjects demonstrated no differences in lumbar proprioception at baseline. Participants from both groups showed better proprioception following the 8-week interval, demonstrating the presence of learning between testing days.

Conclusions: The improvement of lumbar proprioception seen in both groups was ascribed to motor learning of the test itself. The effect of lumbar stabilization exercises on lumbar proprioception remains unknown because the LBP group did not show lumbar proprioception impairments.
\end{abstract}

Keywords: Proprioception, Movement sense, Lumbar spine, Low back pain, Stabilization exercise

\section{Background}

Systematic literature reviews indicate that physical exercises are effective in reducing pain and disability in patients suffering from low back pain (LBP) [1]. Although previous research has not succeeded in showing the superiority of one type of exercise program over another [2], a recent meta-analysis provides more support to strength/resistance and coordination/stabilisation programs [3].

\footnotetext{
* Correspondence: charljea@uqtr.ca

${ }^{1}$ School of Rehabilitation, Faculty of Medicine, Université de Montréal, Montréal, Québec, Canada

${ }^{5}$ Occupational Health and Safety Research Institute Robert-Sauvé (IRSST),

Montréal, Québec, Canada

Full list of author information is available at the end of the article
}

Lumbar coordination/stabilization exercises are designed to restore the neuromuscular control of the lumbar spine. In Panjabi's model of the stabilizing system of the spine [4], an impairment within the passive support system may lead to joint instability, and has to be compensated by the coordinated action of the spinal musculature. Panjabi's model suggests that inadequate lumbar proprioception may contribute to impairments in the passive support system, and may also perpetuate the joint instability, leading to chronic pain [4].

Proprioception can be defined as the sense of stationary position and movement awareness [5]. In the spine, viscoelastic structures and muscles have mechanoreceptors that monitor trunk position and movement [6]. In patients 
with chronic LBP, altered central processing of mechanoreceptor afferent signals may lead to neuromuscular control deficits [7-9]. Paraspinal muscle atrophy [10] and increased fatigability [11] might also represent additional compromises to lumbar stability in patients with LBP. This may explain why some authors suggest adding strength/resistance training following motor control exercises [12]. The effect of such a lumbar stabilization program on lumbar proprioception has never been tested.

The first aim of this study was to determine the effects of an 8-week stabilization exercise program on lumbar proprioception in patients with LBP. A secondary aim was to assess the 8-week test-retest reliability of lumbar proprioception data, with no intervention, in control subjects. The control group was used for pre-treatment comparisons with the patients and to account for possible learning effects from the testing alone. It was hypothesized that patients with LBP would show initial lumbar proprioception impairments, compared to control subjects, that would disappear (or at least be reduced) at the end of the exercise program.

\section{Methods \\ Participants}

Sample size for the reliability study was estimated in order to obtain a target reliability coefficient (ICC) of 0.80 (95\% confidence interval: 0.6-1.0). Based on five experimental trials over two individual testing sessions, a minimum of 28 participants was needed considering the abovementioned requirements [13]. The study conducted by Lee et al. [14] is the only one reporting a statistically significant difference between both groups with regard to MPT in axial rotation, and using 24 patients with LBP and 24 control subjects. Based on the study conducted by Lee et al. [14] the present study have been conservative with a sample size of 29 patients with LBP and 30 control subjects. All participants were aged between 18 and 65 years old, and were recruited through newspaper advertisements and from physiotherapy clinics in Montreal, Quebec, Canada. All participants spoke French and English. Patients with LBP had lumbar or lumbosacral pain, with or without radicular pain, for at least four weeks (non-acute phase), and a score above $12 \%$ on the Oswestry Disability Index (ODI) [15] to allow a minimal important change of $10 \%$ [16] to occur. Patients with non-acute LBP were targeted because exercise is not a primary intervention strategy for acute LBP [1]. General exclusion criteria were: surgery of the pelvis or spinal column; a specific lumbar pathology (fracture, infection or tumor) or scoliosis; systemic or degenerative disease; body mass index over $30 \mathrm{~kg} / \mathrm{m}^{2}$; having begun an exercise program in the last three months; pregnancy and claustrophobia. Additional exclusion criteria for patients with LBP were: having one positive neurological sign in two of three test categories: (1) Achilles and patellar tendon reflexes; (2) Reduced strength in myotomes; (3) Reduced sensation in dermatomes; and litigation relative to the back injury. Exclusion criteria for the control subjects were back pain in the preceding year or a history of back pain lasting more than 1 week.

Before testing, each participant was informed of all experimental procedures and provided their informed written consent. All procedures were approved by the ethics committees of the Centre for Interdisciplinary Research in Rehabilitation of Greater Montreal (CRIR) (ethical registration number: CRIR-738-0512).

\section{Lumbar stabilization program}

An 8-week lumbar stabilization exercise program (two 30-min sessions/week) was provided to the patients with LBP in local physiotherapy clinics, without any cointervention allowed. The patients were encouraged to do the exercises at home. Briefly, the exercise program focused on motor control of the deep trunk muscles [17], followed by gradual inclusion of overloading exercises designed to improve endurance and strength of the paraspinal and abdominal muscles [18] (further details in the Additional file 1).

\section{Questionnaires}

The ODI was used to assess LBP-related disability [15] while the 11-point (0 to 10) numeric pain rating scale (NPRS) was used to assess the current, best and worst levels of pain intensity during the last week, so as to average the three ratings [19].

\section{Lumbar proprioception assessment}

Lumbar motion sense, or motion perception threshold (MPT), was evaluated using a custom built apparatus similar to ones used in previous studies [14, 20] (Fig. 1). The MPT test measured the smallest amount of axial trunk rotation a participant could perceive. This measure was preferred over the measure of lumbar joint position sense because previous findings suggest that this test better discriminates between patients and control subjects [14], and produces more reliable data [20]. During the test, the lumbar spine is passively rotated in the transverse plane (trunk rotation) by rotating the lower body (seat). The seat is positioned on a 16-inches diameter high quality ball bearing (Silverthin Bearing Group, Preston, WA, USA; model SG160CPO) designed to minimize vibration. Movement is driven by a stepper motor (Applied Motion Products, Watsonville, CA, USA; model HT34-504) at a slow, steady rate to minimize tactile cueing. The resolution of the angular measurement was $0.09^{\circ}$.

Participants were positioned in the apparatus so that the L5/S1 joint aligned with the stepper motor shaft. The participant's upper body was secured to the backrest with a 4-point seatbelt to minimize vestibular feedback. Starting 


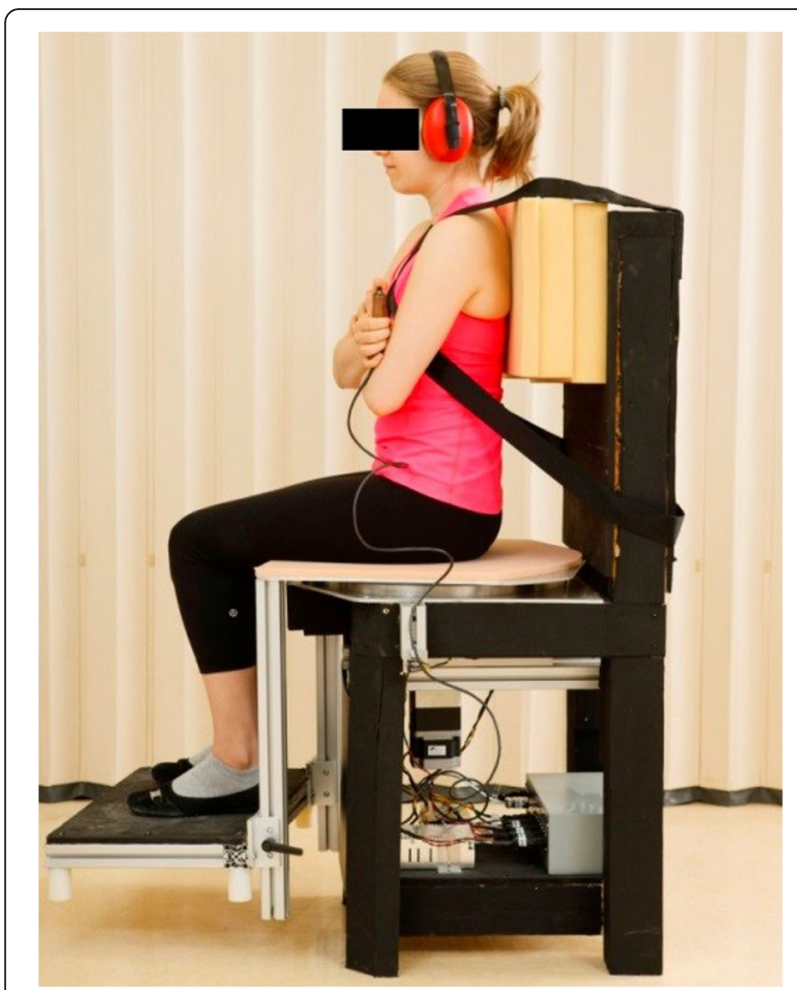

Fig. 1 Motor driven lumbar proprioception chair. The upper body was fixed to the backrest while the lower body (pelvis and lower limbs) was rotated in the transverse plane, inducing an axial rotation of the lumbar spine. In this figure, the lower body is slightly rotated to the right

from the neutral (zero) position, the seat was rotated, either clockwise or counter-clockwise, at a constant rate of $0.2^{\circ} / \mathrm{s}$. As soon as motion was perceived, participants stopped the rotation by pressing a switch, and immediately stated the direction of movement. To remove trials where the subject would have guessed the motion perceptions, the trial was rejected if the direction perceived did not correspond to the true direction [14]. This was done in an attempt to reduce potential noise in the data. Participants were returned to the neutral position following each trial. All trials were performed with eyes closed and noisecanceling headphones.

Prior to testing, participants were given five familiarization trials (or more; until the task was properly understood), randomized for direction. This was followed by ten experimental trials also randomized for direction (five trials per direction).

All testing was done at the start of the study (session 1) and after 8 weeks (session 2).

\section{Home-exercise adherence}

Home-exercise adherence was assessed with one question at the end of the 8-week clinical program: How many times have you done your exercises as prescribed in the last week? The frequency per week was divided by the recommendation of the physiotherapist to obtain a ratio, in accordance with the most common definition of adherence, which is defined as "the extent to which the patient follows medical instructions" [21]. The ratio may vary between 0 and 1 , one being given when the frequency was equal or higher than prescription. The measurement of adherence was not carried out during the course of the 8-week clinical program to avoid influencing positively the adherence behavior (e.g. desirability bias).

\section{Data analysis}

MPT scores were expressed as absolute values, in degrees. No significant differences between left and right rotations were detected, so the data were combined. Outliers (outside the 2-interquartile range) were removed from the $10 \mathrm{MPT}$ scores, to reduce variability. The mean of MPT value, for each participant, was used for analysis.

\section{Reliability statistical analyses}

The reliability of the MPT data from the 30 control subjects was assessed within the generalizability theory framework [22], using a 2-way (2 DAY × 10 TRIALS) ANOVA for repeated measures. The computed sources of variance were used to calculate the dependability coefficients $(\phi)$ and standard error of measurement (SEM) [23]. D-study (decision study) results are reported, based on averaging data from 1,5 and 10 trials within the same testing session (averaging across days is impractical), and may be interpreted in the same manner as an intraclass correlation coefficient [24].

\section{Between-group and between-session statistical analyses}

The healthy subjects whom participated to the reliability study were also used as a "control" group here. This allows testing whether the patients with LBP had proprioceptive deficits at baseline and also allows to estimate if systematic changes that could be detected during the treatment in the patients are attributable to the treatment of to the learning of the measurement protocol. MPT was assessed using a 3-way ANOVA (GROUP: LBP and control subjects; SEX: male and female; DAY: session 1 and 2). Significant interactions or main effects were further analyzed using a post hoc Tukey-Kramer test.

In patients with LBP, clinical outcome measures (NPRS, ODI) were assessed using a 2-way ANOVA (SEX; DAY). Partial Pearson's correlations were also carried out between the change (session 2-session 1) of the MPT measure and the corresponding change of the clinical outcome measures, accounting for the baseline (session 1) MPT measure. 
The analyses above were done with NCSS statistical software (version 8.0 for Windows), with the significance level set at $P<0.05$.

\section{Results}

Preliminary analyses revealed no group (LBP status), SEX or GROUP $\times$ SEX interaction for age, weight or height (Table 1). Among LBP patients, there was no significant SEX effect for NPRS and ODI (Table 1). The duration of pain was distributed as follows across the various intervals proposed [25]: (1) Less than 1 month $(n=0)$, (2) $1-3$ months $(n=2),(3) 3-6$ months $(n=1)$, (4) 6 months- 1 year $(n=4)$, (5) $1-5$ years $(n=10),(6)$ over 5 years $(n=12)$. The sample was thus constituted of $93.1 \%(27 / 29)$ of patients with chronic pain (3 months or more).

\section{Reliability}

For the control group, the 2-way ANOVA detected a DAY effect $(P<0.001)$ (Fig. 2$)$, but no significant TRIAL effect $(P=0.833)$ or interaction $(P=0.663)$. The lack of a TRIAL effect indicates that no within-session learning was present, allowing us to average data across trials to increase reliability.

As expected, the D-study results revealed increased reliability ( $\phi$ increase and SEM decrease) when averaging more trials (Table 2). Averaging 10 trials produced moderately reliable data $\left(\phi=0.59\right.$ and $\left.\mathrm{SEM}=1.2^{\circ}\right)$.

For the between-groups comparison (above), seven, eight, nine or ten trials were averaged per session (representing 2, 8,18 and $72 \%$ of the testing sessions, respectively), after having rejected the outliers. Consequently, the data used in the between-groups comparison are likely to be moderately reliable.

\section{Effects of the stabilization exercise program and between-group comparisons}

All patients with LBP have undertaken the 8-week exercise program and reached the third (final) phase of the exercise program. Home-exercise adherence

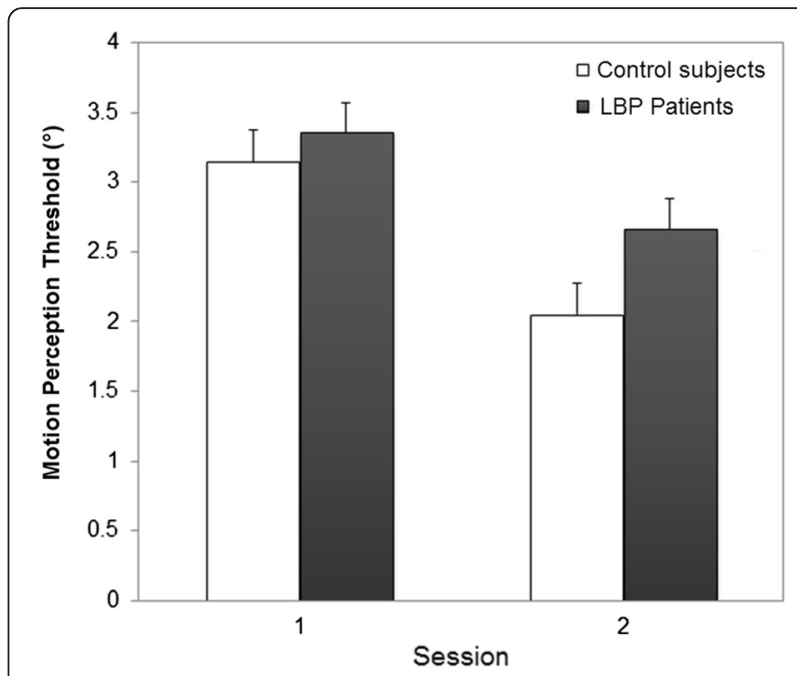

Fig. 2 Averaged (across individuals) motion perception threshold (MPT) for both group in each testing session (mean $\pm \mathrm{SD}$ )

measured at post-treatment was high, with a mean ratio of 0.86 (SD 0.21). For MPT, the only significant finding was a main effect of DAY $(P<0.001)$ (Table 3$)$, showing significantly smaller MPT values at the second testing session (Fig. 2). The lack of a GROUP $\times$ DAY interaction reveals that the change in MPT measures over the 8-week period was not significantly different between the two groups. Figure 3 shows MPT scores of each participant for both testing sessions. The variability of the scores and variability of effects (time/treatment) were similar between groups.

A significant effect of DAY was also found for the clinical measures (NPRS, ODI) in patients with LBP, showing a decrease of pain and disability after the stabilization program (Table 3; Figs. 4 and 5). No significant SEX $\times$ DAY or SEX effects were found.

Partial correlation analyses revealed no significant correlations between changes in MPT scores and changes in NPRS $(\mathrm{r}=-0.06 ; P=0.744)$ or ODI $(r=-0.10 ; P=0.602)$.

Table 1 Demographic and clinical profiles [Mean (SD)] of the participants at initial measurements session (Day 1) according to their sex and clinical conditions

\begin{tabular}{|c|c|c|c|c|c|c|c|}
\hline \multirow[b]{3}{*}{ Variable } & \multicolumn{2}{|c|}{ Control subjects } & \multicolumn{2}{|c|}{ LBP patients } & \multicolumn{3}{|c|}{$P$ values } \\
\hline & Men & Women & Men & Women & Group & Sex & Group $\times$ Sex \\
\hline & $(n=15)$ & $(n=15)$ & $(n=14)$ & $(n=15)$ & & & \\
\hline Age (years) & $39.3(14.3)$ & $39.8(13.7)$ & $43.5(13.3)$ & $47.3(12.0)$ & 0.093 & 0.424 & 0.620 \\
\hline Height (cm) & $178.0(8.4)$ & $164.2(6.1)$ & $172.7(5.9)$ & $163.6(5.3)$ & 0.080 & 0.124 & 0.163 \\
\hline Weight (kg) & $77.1(10.7)$ & $62.9(10.9)$ & 76.0 (13.3) & $72.2(9.6)$ & 0.154 & 0.331 & 0.069 \\
\hline BMI $\left(\mathrm{kg} / \mathrm{m}^{2}\right)$ & $24.4(3.2)$ & $23.3(3.6)$ & 25.7 (3.8) & $25.4(3.4)$ & 0.063 & 0.466 & 0.249 \\
\hline NPRS (/10) & & & $3.0(2.2)$ & $3.6(1.5)$ & & 0.388 & \\
\hline ODI (\%) & & & $27.9(9.2)$ & $30.0(9.8)$ & & 0.518 & \\
\hline
\end{tabular}


Table 2 Reliability results (D-Study)

\begin{tabular}{|c|c|c|c|c|c|c|c|c|c|c|c|}
\hline \multirow[t]{2}{*}{ Variable } & \multirow{2}{*}{$\begin{array}{l}\text { Mean } \\
(\mathrm{SD})\end{array}$} & \multicolumn{3}{|c|}{ Strategy: 1 trial/day } & \multicolumn{3}{|c|}{ Strategy: 5 trials/day } & \multicolumn{3}{|c|}{ Strategy: 10 trials/day } & \multirow{2}{*}{$\begin{array}{l}\mathrm{N}^{\mathrm{a}} \\
\varphi>0.75\end{array}$} \\
\hline & & $\varphi$ & SEM & SEM(\%) & $\varphi$ & SEM & SEM(\%) & $\varphi$ & SEM & SEM(\%) & \\
\hline MPT & $2.6(2.2)$ & 0.43 & 1.7 & 66 & 0.57 & 1.3 & 50 & 0.59 & 1.2 & 47 & $\infty$ \\
\hline
\end{tabular}

SD Standard Deviation, $\varphi$ Index of dependability, SEM standard error of measurement, SEM (\%) SEM expressed in percentage of the grand mean; ${ }^{\text {a }}$ Number of trials required to reach $\varphi \geq 0.75, \infty$ indicating when it is impossible to reach this threshold

\section{Discussion}

The primary aim of the present study was to assess the effects of an 8-week stabilization exercise program on the lumbar MPT in patients with LBP. While the patients showed a lower MPT at the end ot the stabilization program, a similar effect was found in control subjects, for whom no treatment was provided. In fact, no betweengroup differences were found. In line with these findings, no significant correlation was found between the change of MPT and the change of LBP intensity (NPRS) or LBPrelated disability (ODI).

The secondary aim of the study was to assess the 8week test-retest reliability of MPT in control subjects. The reliability was moderate, and illustrated the presence of a systematic learning effect between testing sessions.

Before discussing these findings, it is important to explain why the MPT scores reported here are much higher than the scores of the studies that have used a similar protocol and device $[14,20]$, the averaged scores (across individuals) ranging between 1.1 and $1.4^{\circ}$ in these studies. In these studies, the two worst trials (highest scores) were removed from their 8 to 10 trials available, while in the present study, only outliers (worst or best scores) were removed. This may at least partly explain our higher averaged scores, the number of practice trials being approximately the same.

\section{8-week test-retest reliability}

The present reliability findings are the first attempt to assess the reliability of MPT data over the duration of a rehabilitation program (8 weeks). Two previous studies

Table 3 Statistical results ( $P$ values) corresponding to the comparisons between control subjects and patients with LBP (GROUP factor), between men and women (SEX factor), between measurement sessions (DAY factor) and the corresponding interactions

\begin{tabular}{llllllll}
\hline Variable & \multicolumn{6}{l}{ ANOVA results ( $P$ values) } \\
\cline { 2 - 8 } & GROUP & SEX & DAY & $G \times S$ & $G \times D$ & $S \times D$ & $G \times S \times D$ \\
& $(G)$ & $(\mathrm{S})$ & $(\mathrm{D})$ & & & & \\
\hline NPRS & $/$ & 0.641 & $<0.001$ & $/$ & $/$ & 0.513 & $/$ \\
ODI & $/$ & 0.429 & $<0.001$ & $/$ & $/$ & 0.547 & $/$ \\
MPT & 0.239 & 0.509 & $<0.001$ & 0.303 & 0.271 & 0.253 & 0.088
\end{tabular}

NPRS numeric pain rating scale, ODI Oswestry Disability Index, MPT motion perception threshold; "/" = not applicable because the corresponding ANOVA model did not include a GROUP factor have reported reliability results for MPT measures taken with a device similar to the one used in the present study $[8,20]$. The reliability of these previous data were high (ICCs over 0.77; SEM: $0.34^{\circ}$ ), but only permitted a within-day reliability analysis. The lower reliability of our data are at least partly explained by an apparent long-term learning effect (main effect of DAY), which suggest the presence of motor learning. The familiarization trials that were used at the beginning of each day were apparently good enough to eliminate possible within-session learning (no TRIAL main effect), but insufficient to eliminate between-day learning, the latter being likely explained by the effect of sleep on neural plasticity [26]. This is worth mentioning that the subject needs to be well concentrated because the chair movement is very difficult to detect. Introducing a familiarization day before the first testing session (double baseline measurement) might help to minimize the long-term learning effect.

\section{Effect of 8-week stabilization exercise program on MPT}

To the authors' knowledge, this is the first study to assess the effects of an 8-week stabilization exercise program on MPT. While the patients in the study did show significant improvements in MPT following the exercise program, their improvement was comparable to that of a healthy control group who received no intervention. This suggests that patients' improvements in MPT may be more attributable to having learned the testing procedure than to the exercise program. Consequently, it is not surprising that we observed no correlation between the change of MPT and the change in the clinical outcomes (NPRS, ODI).

One explanation for the apparent lack of a MPT training effect is that the stabilization program used in this study is not specifically designed to improve lumbar proprioception. These exercises do, however, include some required components (coordination, muscle performance and balance training) to improve proprioception [27]. These results may also be related to the fact that the patients with LBP in this study did not show MPT impairments at the beginning of the program, relative to the control group. It is also possible that different findings would have emerged if we had used a measure of active joint position sense, rather than passive MPT. Lumbar stabilization exercises may provide a better training stimulus for proprioception in situations in 

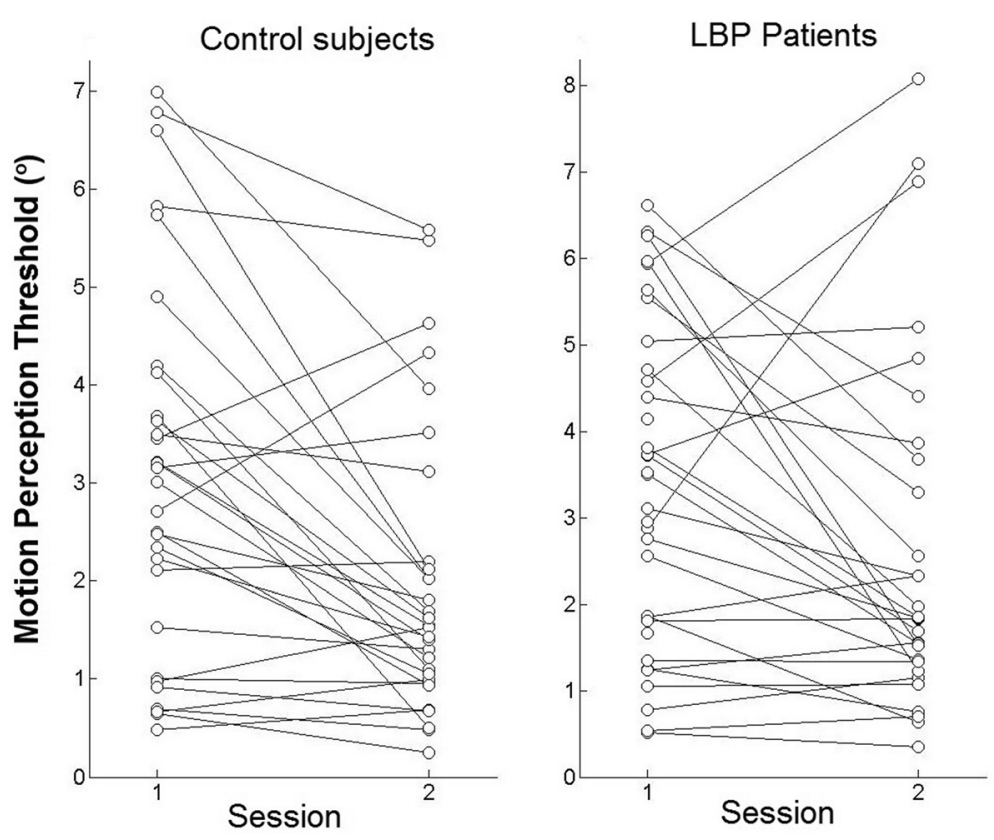

Fig. 3 Line graph representing MPT scores of all participants in each group for both testing sessions

which the trunk muscles are involved in maintaining lumbar stability.

\section{Between-group comparisons on MPT}

There is still considerable discrepancy in the literature with regards to impairment of trunk proprioception in patients with LBP [20]. Considering that fundamental differences exist in the different available proprioception measures (MPT, active and passive joint position sense, force sense) [28], we will focus on MPT findings in the transverse plane (lumbar spine rotation) $[8,14,20]$.

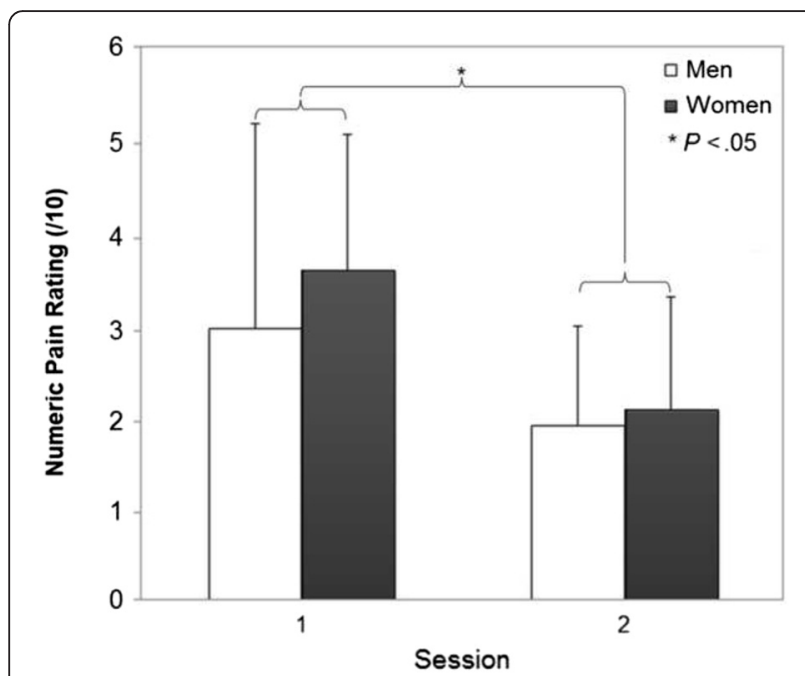

Fig. 4 Numeric pain rating scale (NPRS) for each sex and testing day $($ mean $\pm \mathrm{SD})$
Previous studies have compared MPT between LBP patients and control subjects, using a device similar to the one used in the present study. Two studies showed significantly higher MPT in patients with LBP $[8,14]$, while one study showed no difference as in the present study [20]. However, the study conducted by Selfies et al. [20] involved young athletes with history of low back injury at the start of the study, which differ from patients with LBP in the present study. As patients with non-specific LBP are a very heterogeneous group, such conflicting results

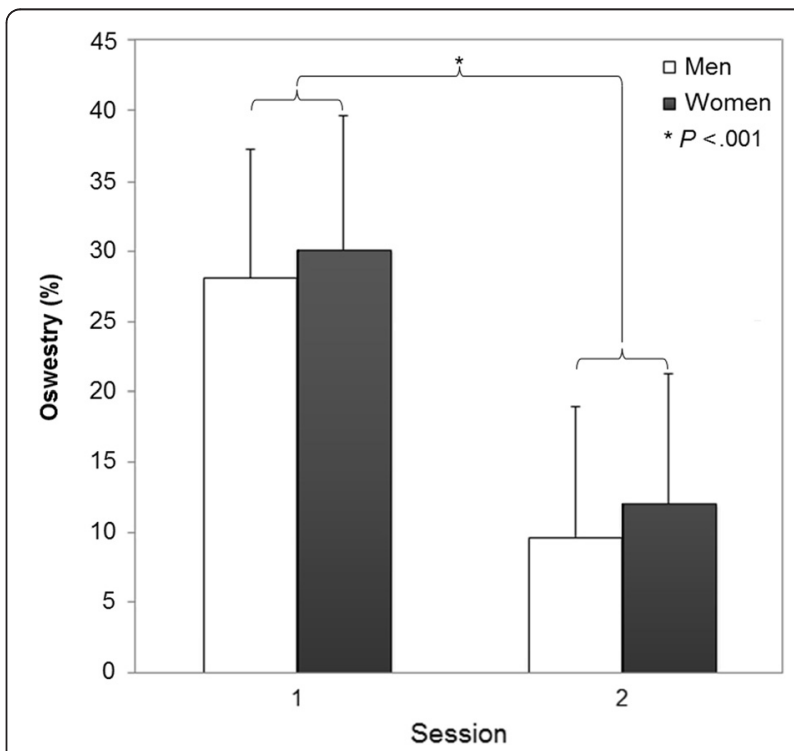

Fig. 5 Oswestry (ODI) disability scores for each sex and testing day (mean $\pm \mathrm{SD})$ 
are not surprising. Unfortunately, the prevalence of patients with a clinically relevant proprioceptive deficit has never been substantiated with a representative sample of patients. Consequently, the number of patients required to study clinically-relevant subgroups of patients, based on their level of proprioceptive deficits, is unknown. Heterogeneity of MPT scores was not higher in the patient group, comparatively to the control group (Fig. 3), as the change of scores over time. Consequently, the presence of clinical subgroups is not obvious within this small sample of patients. The two studies that looked at sex comparisons showed no difference in position sense $[8,20]$, which concurs with the present findings.

\section{Limitations}

The test-retest reliability results are limited to healthy subjects. Patients with low back pain could have produced different findings. The healthy "control group" used in the present study does not correspond to a conventional control group composed of patients that have not received any treatment. Consequently, although unlikely, it would be possible that such a patient-based control group would have shown no learning of the motion perception measurement, which in turn would have enhanced the likelihood of detecting a GROUP $\times$ DAY interaction. As patients with non-specific LBP are a very heterogeneous group, a larger sample of patients is needed to explore possible clinical subgroups based on their level of proprioceptive deficits. This would have been valuable to study relationships with changes in clinical outcomes over treatment. There was no blinding in the assessment to which group the participant was in (LBP patient or control subject), although this type of objective assessment is not believed to be affected by the experimenter. Finally, a single baseline measurement was carried out. Future studies should considered multiple baseline measurements in lumbar MPT in order to wash-out learning effects to better isolate treatment effects.

\section{Conclusion}

Patients with LBP did not show lumbar MPT impairments in the present study, which likely explain why they didn't improve their proprioception over an 8-week stabilization exercise program, when compared with the changes shown by a healthy control group that not received any treatment. The presence in our data of a long-term learning effect in MPT, with repeated testing, highlights the need to better determine the measurement properties (reliability, validity, responsiveness) of tools used to assess the effects of lumbar stabilization programs.

\section{Additional file}

Additional file 1: Description of the 8-week lumbar stabilization exercise program. (PDF $901 \mathrm{~kb}$ )

\section{Competing interests}

The authors declare that they have no competing interests.

\section{Authors' contributions}

All authors played a significant role in this project; RP, SMH, J-PD and CL worked together to gain ethical approval and assisted with gaining funding to conduct this research. All contributed to the research design. J-AB and CL processed and analyzed the data, and wrote the paper. Finally, all authors read and approved the final manuscript.

\section{Acknowledgements}

The present research project was funded (\#2010-0022) by the Occupational Health and Safety Research Institute Robert-Sauvé (IRSST) of Quebec (Canada). Thanks go to Sophie Bellefeuille, Marilee Nugent and Cynthia Appleby for data collection and to Hakim Mecheri for engineering assistance. Finally, special thanks go to Jacek Cholewicki, Peter Reeves and Angela Lee who shared the design of the proprioception chair.

\section{Author details}

${ }^{1}$ School of Rehabilitation, Faculty of Medicine, Université de Montréal, Montréal, Québec, Canada. ${ }^{2}$ School of Physical \& Occupational Therapy, McGill University, Montréal, Québec, Canada. ${ }^{3}$ Deparment of Rehabilitation and Movement Science, The University of Vermont, Burlington, Vermont, USA. ${ }^{4}$ School of Rehabilitation, Faculty of Medicine, Université de Sherbrooke, Sherbrooke, Québec, Canada. ${ }^{5}$ Occupational Health and Safety Research Institute Robert-Sauvé (IRSST), Montréal, Québec, Canada. ${ }^{6}$ Center for Interdisciplinary Research in Rehabilitation of Greater Montreal (CRIR), Montreal Rehabilitation Institute, Montreal, Québec, Canada.

Received: 12 September 2015 Accepted: 6 January 2016

Published online: 14 January 2016

\section{References}

1. Smidt N, de Vet HC, Bouter LM, Dekker J, Arendzen JH, de Bie RA, et al. Effectiveness of exercise therapy: a best-evidence summary of systematic reviews. Aust J Physiother. 2005:51:71-85.

2. Hayden JA, van Tulder MW, Tomlinson G. Systematic review: strategies for using exercise therapy to improve outcomes in chronic low back pain. Ann Intern Med. 2005;142:776-85.

3. Searle A, Spink M, Ho A, Chuter V. Exercise interventions for the treatment of chronic low back pain: A systematic review and meta-analysis of randomised controlled trials. Clin Rehabil. 2015:29:1155-67.

4. Panjabi MM. A hypothesis of chronic low back pain: ligament subfailure in juries lead to muscle control dysfunction. Eur Spine J. 2006;15:668-76.

5. Riemann BL, Lephart SM. The sensorimotor system, Part I: The physiologic basis of functional joint stability. J Athl Train. 2002;37:71-9.

6. Holm S, Indahl A, Solomonow M. Sensorimotor control of the spine. J Electromyogr Kinesiol. 2002;12:219-34.

7. Brumagne S, Cordo P, Lysens R, Verschueren S, Swinnen S. The role of paraspinal muscle spindles in lumbosacral position sense in individuals with and without low back pain. Spine. 2000;25:989-94.

8. Taimela S, Kankaanpaa M, Luoto S. The effect of lumbar fatigue on the ability to sense a change in lumbar position. A controlled study. Spine. 1999;24:1322-7.

9. Solomonov M. Sensory-motor control of ligaments and associated neuromuscular disorders. J Electromyogr Kinesiol. 2006;16:549-67.

10. Danneels LA, Vanderstraeten GG, Cambier DC, Witvrouw EE, De Cuyper HJ. CT imaging of trunk muscles in chronic low back pain patients and healthy control subjects. Eur Spine J. 2000;9:266-72.

11. Lariviere C, Bilodeau M, Forget R, Vadeboncoeur R, Mecheri H. Poor back muscle endurance is related to pain catastrophizing in patients with chronic low back pain. Spine. 2010;35:E1178-1186.

12. Koumantakis GA, Watson PJ, Oldham JA. Trunk muscle stabilization training plus general exercise versus general exercise only: randomized controlled trial of patients with recurrent low back pain. Phys Ther. 2005;85:209-25. 
13. Shoukri MM. Sample size requirements for the design of reliability study: review and results. Stat Methods Med Res. 2004;13:251-71.

14. Lee AS, Cholewicki J, Reeves NP, Zazulak BT, Mysliwiec LW. Comparison of trunk proprioception between patients with low back pain and healthy controls. Arch Phys Med Rehabil. 2010;91:1327-31.

15. Vogler D, Paillex R, Norberg M, de Goumoens P, Cabri J. Cross-cultural validation of the Oswestry disability index in French. Ann Readapt Med Phys. 2008:51:379-85.

16. Ostelo RW, Deyo RA, Stratford P, Waddell G, Croft P, Von Korff M, et al. Interpreting change scores for pain and functional status in low back pain: towards international consensus regarding minimal important change. Spine. 2008;33:90-4.

17. Richardson C, Hodges PW, Hides JA. Therapeutic exercise for lumbopelvic stabilization: a motor control approach for the treatment and prevention of low back pain. In., 2nd edn. Sydney: 2nd ed. London: Churchill Livingstone; 2004.

18. McGill S. Low Back Disorders. Evidence-based prevention and rehabilitation. 2nd ed. Champaign: Human Kinetics; 2002. p. 328.

19. Childs JD, Piva SR, Fritz JM. Responsiveness of the numeric pain rating scale in patients with low back pain. Spine. 2005;30:1331-4.

20. Silfies SP, Cholewicki J, Reeves NP, Greene HS. Lumbar position sense and the risk of low back injuries in college athletes: a prospective cohort study. BMC Musculoskelet Disord. 2007;8:129

21. Haynes RB, Taylor WD, Sackett DL. Compliance in health care. Baltimore: Johns Hopkins University Press; 1979.

22. Shavelson RJ, Webb NM. Generalizability theory. A primer. London: SAGE Publications; 1991.

23. Santos BR, Delisle A, Larivière C, Plamondon A, Imbeau D. Reliability of center of pressure summary measures of postural steadiness in healthy young adults. Gait Posture. 2008;27:408-15.

24. Fleiss RL. The design and analysis of clinical experiments. New York: John Wiley and Sons; 1986.

25. Deyo RA, Dworkin SF, Amtmann D, Andersson G, Borenstein D, Carragee E, et al. Report of the NIH Task Force on Research Standards for Chronic Low Back Pain. Phys Ther. 2015;95:e1-e18.

26. Gorgoni M, D'Atri A, Lauri G, Rossini PM, Ferlazzo F, De Gennaro L. Is sleep essential for neural plasticity in humans, and how does it affect motor and cognitive recovery? Neural Plast. 2013;3013:13.

27. Clark NC, Röijezen U, Treleaven J. Proprioception in musculoskeletal rehabilitation. Part 2: Clinical assessment and intervention. Man Ther. 2015;20:378-87.

28. Röijezen U, Clark NC, Treleaven J. Proprioception in musculoskeletal rehabiliation. Part 1: Basic science and principles of assessment and clinical interventions. Man Ther. 2015:20:368-77.

\section{Submit your next manuscript to BioMed Central and we will help you at every step:}

- We accept pre-submission inquiries

- Our selector tool helps you to find the most relevant journal

- We provide round the clock customer support

- Convenient online submission

- Thorough peer review

- Inclusion in PubMed and all major indexing services

- Maximum visibility for your research

Submit your manuscript at www.biomedcentral.com/submit

) Biomed Central 